\title{
INDICADORES ANTROPOMÉTRICOS DE ESTADO NUTRICIONAL COMO PREDITORES DE CAPACIDADE EM IDOSOS
}

\author{
ANTHROPOMETRIC INDICATORS OF NUTRITIONAL STATUS AS A PREDICTOR OF \\ CAPACITY IN THE ELDERLY \\ LOS INDICADORES ANTROPOMÉTRICOS DEL ESTADO NUTRICIONAL COMO \\ FACTOR PREDICTIVO DE CAPACIDAD EN EL ANCIANO
}

Artigo Original

Kleyton Trindade Santos ${ }^{1}$

(Fisioterapeuta)

José Carlos Cândido dos Santos

Júnior ${ }^{1}$ (Fisioterapeuta)

Saulo Vasconcelos Rocha'

(Educador Físico)

Luciana Araújo dos Reis'

(Fisioterapeuta)

Raildo da Silva Coqueiro ${ }^{1}$

(Educador Físico)

Marcos Henrique Fernandes ${ }^{1}$

(Fisioterapeuta)

1. Universidade Estadual do Sudoeste da Bahia, Jequié, BA, Brasil.

\section{Correspondência:}

Marcos Henrique Fernandes, Departamento de Saúde, Núcleo de Estudos em Epidemiologia do Envelhecimento, Universidade Estadual do Sudoeste da Bahia. Av. José Moreira Sobrinho, s/n, Jequiezinho, 45206 - 190 Jequié, BA, Brasil.

marcoshenriquefernandes@bol.com.br

\section{RESUMO}

Introdução: Trata-se de estudo seccional que analisou dados de pesquisa epidemiológica de base populacional e domiciliar conduzida em um município do nordeste do Brasil. Objetivo: Identificar os indicadores antropométricos de estado nutricional que apresentam melhor capacidade preditiva de capacidade funcional em idosos. Métodos: A população foi constituída por 316 idosos que foram avaliados por meio de questionário contendo informações sociodemográficas, atividade física e condições de saúde, seguido de avaliação antropométrica. A capacidade funcional foi avaliada por meio das escalas de Katz e de Lawton e Brody para atividades básicas e instrumentais da vida diária, respectivamente. Foram utilizados como indicadores de estado nutricional, o índice de massa corporal, a área muscular do braço e o perímetro da panturrilha. Estimou-se modelos simples e múltiplos de regressão logística multinomial, tendo a capacidade funcional como variável dependente e os indicadores antropométricos como variáveis de interesse. Resultados: Participaram do estudo 173 mulheres (54,7\%) e 143 homens (45,3\%). Os resultados da regressão múltipla mostraram que o perímetro da panturrilha foi o único preditor de capacidade funcional em mulheres $\left(\leq P_{25}\right.$ : $O R=5,77, p=0,028$, para dependência nas atividades instrumentais), enquanto que nos homens o índice de massa corporal foi o único indicador associado (baixo peso: $O R=11,36, p=0,006$; sobrepeso: $O R=22,06, p=0,002$; para dependência nas atividades básicas e instrumentais). Conclusão: Os resultados permitem concluir que os indicadores antropométricos preditores de capacidade funcional em idosos variam de acordo com o sexo, com o perímetro da panturilha sendo mais adequada às mulheres e o índice de massa corporal aos homens.

Palavras-chave: antropometria, envelhecimento, estado nutricional, idoso fragilizado, saúde do idoso.

\section{ABSTRACT}

Introduction: It is a sectional study which analyzed data from a population-based household epidemiological study conducted in a municipality in northeastern Brazil. Objective: Identify anthropometric indicators of nutritional status which have better predictive ability of functional capacity in the elderly. Methods: The population consisted of 316 elderly people who were assessed by a questionnaire containing sociodemographic information, physical activity and health conditions, followed by anthropometric evaluations. Functional capacity was assessed using Katz's basic activities of daily living (ADL) and Lawton-Brody's Instrumental Activities of Daily Living (IADL) scales, respectively. Body mass index, arm muscle area and calf perimeter were used as indicators of nutritional status. We estimated simple and multiple models of multinomial logistic regression, considering functional capacity as the dependent variable and the anthropometric indicators as variables of interest. Results: The study included 173 women (54.7\%) and 143 men (45.3\%). The multiple regression results showed that the calf perimeter was the only predictor of functional capacity in women ( $\leq P_{25}: O R=5.77, p=0.028$, for dependence in instrumental activities), while in men the body mass index was the only associated indicator (underweight: $O R=11.36, p=0.006$; overweight: $O R=22.06, p$ $=0.002$ for dependence in basic and instrumental activities). Conclusion: The results suggest that anthropometric predictors of functional capacity in the elderly vary according to gender, with the calf perimeter being more suitable for women and the body mass index for men.

Keywords: anthropometry, aging, nutritional status, frail elderly, health of the elderly.

\section{RESUMEN}

Introducción: Se trata de estudio seccional que analizó datos de encuesta epidemiológica de base poblacional y domiciliaria conducida en un municipio del noreste de Brasil. Objetivo: Identificar los indicadores antropométricos de estado nutricional que presentan mejor capacidad predictiva de capacidad funcional en ancianos. Métodos: La población fue constituida por 316 ancianos que fueron evaluados por medio de cuestionario conteniendo informaciones sociodemográficas, actividad física y condiciones de salud, seguido de evaluación antropométrica. La capacidad funcional fue evaluada por medio de las escalas de Katz y de Lawton y Brody para actividades básicas e instrumentales de la vida diaria, respectivamente. Fueron utilizados como indicadores 
de estado nutricional, el índice de masa corporal, el área muscular del brazo y el perímetro de la pantorrilla. Se estimaron modelos simples y múltiples de regresión logística multinomial, teniendo la capacidad funcional como variable dependiente y los indicadores antropométricos como variables de interés. Resultados: Participaron en el estudio 173 mujeres $(54,7 \%)$ y 143 hombres (45,3\%). Los resultados de la regresión múltiple mostraron que el perímetro de la pantorrilla fue el único predictor de capacidad funcional en mujeres $\left(\leq P_{25}: O R=5,77, p=0,028\right.$, para dependencia en las actividades instrumentales), mientras que en los hombres el indice de masa corporal fue el único indicador asociado (bajo peso: $O R=11,36, p=0,006$; sobrepeso: $O R=22,06, p=0,002$; para dependencia en las actividades básicas e instrumentales). Conclusión: Los resultados permiten concluir que los indicadores antropométricos predictores de capacidad funcional en ancianos varían de acuerdo con el sexo, con el perímetro de la pantorrilla siendo más adecuada a las mujeres y el índice de masa corporal a los hombres.

Palabras clave: antropometría, envejecimiento, estado nutricional, anciano fragilizado, salud del anciano.

\section{INTRODUÇÃO}

O crescimento da população idosa é um fenômeno mundial e, no Brasil, as mudanças estão ocorrendo de forma radical e muito rápida. Nos países em desenvolvimento, como o Brasil, esse processo não ocorre concomitantemente com as mudanças na atenção a saúde, favorecendo situações onde idosos sevapresentam com prejuízo nutricional e incapacidades para realizações de suas tarefas?

A presença de risco nutricional elevado tende ao declínio acentuado no desempenho físico ${ }^{2}$. Dessa forma, o estado nutricional pode interferir nas condições dos indivíduos de realizar as atividades da vida diária de forma independente, autônoma e satisfatória. Fatores como níveis de força e resistência musculares, fator nutricional, manutenção da flexibilidade, motricidade e comorbidade no idoso influenciam na capacidade funcional ${ }^{3}$.

Diversos são os indicadores antropométricos disponíveis para avaliação do estado nutricional em idosos; a maioria deles apresenta como vantagens facilidade de obtenção, baixo custo e pequena variação intra e interavaliadores ${ }^{4}$. Embora o índice de massa corporal (IMC) seja o indicador mais comumente utilizado ${ }^{5}$, uma das dificuldades na avaliação geriátrica é a definição do melhor marcador de risco a saúde. Apesar de estar estabelecido que a função física esteja intimamente ligada ao estado nutricional², existe carência de pesquisas populacionais que avaliem de forma comparativa a capacidade preditiva de indicadores antropométricos em idosos residentes em comunidade, a fim de se identificar o melhor preditor de capacidade funcional.

Diante do exposto, o objetivo do estudo é identificar os indicadores antropométricos de estado nutricional que apresenta melhor capacidade preditiva de capacidade funcional em idosos.

\section{MÉTODOS}

Trata-se de um estudo seccional que analisou dados de uma pesquisa epidemiológica de base domiciliar, denominada "Estado nutricional, comportamentos de risco e condições de saúde dos idosos de Lafaiete Coutinho-BA".

Um censo foi conduzido na cidade de Lafaiete Coutinho, BA, Brasil, em Janeiro de 2011 para a identificação dos idosos participantes da pesquisa. Todos os residentes na zona urbana com idade $\geq 60$ anos (n = 355) foram procurados para entrevistas e exames (testes sanguíneos, aferição da pressão arterial, medidas antropométricas e testes motores). A localização das residências foi feita por meio das informações da ESF. Dos 355 participantes que compunham a população idosa do município, participaram da pesquisa 316 (89,0\%); foram registradas 17 recusas $(4,8 \%)$ e $22(6,2 \%)$ indivíduos que não foram localizados após três visitas domiciliares em dias alternados, sendo considerados perdas.
Foi utilizado um formulário próprio, baseado no questionário usado na Pesquisa Saúde, Bem Estar e Envelhecimento - SABE - em sete países da América Latina e Caribe, ${ }^{6}$ a exceção dos questionários de atividade física e consumo alimentar que, no presente estudo, foram mais amplos.

As seguintes informações foram usadas neste estudo: (1) antropometria [massa corporal, estatura, perímetro da panturrilha, perímetro do braço e dobra cutânea tricipital (DCT)]; (2) capacidade funcional; (3) Atividade física habitual; (4) características sócio-demográficas (idade, sexo, sabe ler e escrever, e renda familiar per capita); (5) condições de saúde (doenças crônicas, queda e hospitalização) e (6) tabagismo.

O protocolo do estudo foi aprovado pelo Comitê de Ética em Pesquisa da Universidade Estadual do Sudoeste da Bahia, BA, Brasil, (n 064/2010). A participação foi voluntária, e todos os indivíduos assinaram o termo de consentimento livre e esclarecido.

\section{Capacidade funcional (variável dependente)}

Para avaliar a capacidade funcional foram utilizadas as informações do instrumento de Katz et al. ${ }^{7}$, para atividades básicas da vida diária - ABVDs - (tomar banho, alimenta-se, deitar e levantar da cama, ir ao banheiro, vestir-se e controlar esfíncteres) e da escala de Lawton e Brody ${ }^{8}$ para atividades instrumentais da vida diária (AIVDs) (preparar uma refeição quente, cuidar do próprio dinheiro, ir a lugares sozinho, fazer compras, telefonar, fazer tarefas domésticas leves, fazer tarefas domésticas pesadas e tomar medicamentos).

$\mathrm{Na}$ entrevista os participantes foram interrogados sobre a presença de dificuldades ou necessidade de ajuda com cada atividade. Os idosos foram classificados como independentes caso não relatassem necessidade de ajuda para realizar alguma ABVD e AIVD, e dependentes quando relatou necessidade de ajuda em, pelo menos, uma das atividades de cada dimensão. Conforme proposto por Hoyemans et al ${ }^{9}$, foi construída uma escala de incapacidade funcional hierárquica distinguindo três categorias: (1) independentes, (2) dependentes nas AIVDs, (3) dependentes nas ABVDs e AIVDs. Os idosos que relataram dependência nas ABVDs, mas não nas atividades instrumentais, foram classificados na última categoria, referentes à dependência em ambas as dimensões.

Optou-se por avaliar a capacidade funcional pelas escalas de Katz e Lawton 7,8 , através do auto-relato e considerando a dependência. A primeira escala é o indicador mais utilizado na avaliação da capacidade funcional, considerando que em ambos os instrumentos não se refere apenas à dimensão física, permitindo uma visão ampla do indivíduo ${ }^{10}$. 


\section{Indicadores antropométricos de estado nutricional (variáveis explanatórias)}

A massa corporal foi medida com balança digital portátil (Zhongshan Camry Eletronic, G-Tech Glass 6, China), com o indivíduo descalço e usando o mínimo de roupas possível. A estatura foi mensurada de acordo com a técnica de Frisancho ${ }^{11}$, utilizando um estadiômetro compacto portátil (Wiso, China) instalado em local adequado, segundo as normas do fabricante. O perímetro do braço foi medido com uma trena antropométrica inelástica (ABN ${ }^{\top M}$, Brasil) conforme Callaway et al. ${ }^{12}$. A DCT foi mensurada com compasso de dobras cutâneas (WCS, Brasil) de acordo com Harrison et al. ${ }^{13}$. Todas as medidas antropométricas, exceto a massa corporal, foram realizadas em triplicata, e os valores médios foram utilizados nas análises. $\mathrm{O}$ índice de massa corporal [IMC = massa corporal $(\mathrm{kg}) /$ estatuta $\left.^{2}(\mathrm{~m})\right]$ e a área muscular do braço $\{\mathrm{AMB}=[(\mathrm{CB}-\pi$ $\left.\times D C T)^{2} / 4 \times \pi\right]-10$, para homens; $A M B=\left[(C B-\pi \times D C T)^{2} / 4 \times \pi\right]-6,5$, para mulheres\} foram calculados ${ }^{14}$.

O IMC foi classificado da seguinte forma ${ }^{15}:<22,0 \mathrm{~kg} / \mathrm{m}^{2}$, baixo peso; $22,0 \leq \mathrm{IMC} \leq 27,0 \mathrm{~kg} / \mathrm{m}^{2}$, adequado; $>27,0 \mathrm{~kg} / \mathrm{m}^{2}$, sobrepeso. A AMB e o perímetro da panturrilha foram categorizados com base nos percentis $\left(P_{k}\right)$, de acordo com o sexo: $\leq P_{25}$ : inferior; $>P_{25}$ a $\leq P_{75}$ :médio; $>P_{75:}$ superior.

\section{Variáveis de ajuste}

Foram utilizados como variáveis de ajuste: idade (como variável contínua); número de doenças crônicas (nenhuma, uma e duas ou mais) considerando-se hipertensão, diabetes, câncer (exceto tumores na pele), doença crônica do pulmão, problemas cardíacos, circulatórios, reumáticos e osteoporose; hospitalização nos últimos 12 meses (nenhuma e uma ou mais vezes); queda nos últimos 12 meses (sim e não); tabagismo (fumante, ex-fumante e nunca fumou); atividade física habitual, avaliada através do International Physical Activity Questionnaire (IPAQ), versão longa ${ }^{16}$ (insuficientemente ativo: menor que 150 minutos por semana em atividades físicas moderadas ou vigorosas e ativo: maior ou igual a 150 minutos por semana) $)^{17}$.

As variáveis de ajuste foram selecionadas em conformidade com outros estudos que mostraram relações dessas variáveis com estado nutricional e capacidade funcional18,19.

\section{Procedimento estatístico}

Foram estimados modelos simples e múltiplos de regressão logística multinomial, tendo a capacidade funcional como variável dependente e o IMC, AMB e perímetro da panturrilha como variáveis de interesse. A categoria independente de capacidade funcional foi definida como o grupo de referência.

Em todas as análises o nível de significância de $5 \%(a=0,05)$ foi usado para estimar os intervalos de confiança de 95\% (IC95\%) para a odds ratios (OR). Os dados foram tabulados e analisados no The Statistical Package for Social Sciences para Windows (SPSS. 15.0, 2006, SPSS, Inc, Chicago, IL).

\section{RESULTADOS}

Participaram do estudo 173 mulheres $(54,7 \%)$ e 143 homens (45,3\%). A idade variou de 60 a 105 anos, com a média de 74,2 $\pm 9,8$ anos. A média de idade foi de $74,9 \pm 10$ anos (60 - 103) nas mulheres e de 73,4 \pm 9,4 anos nos homens (faixa etária?). Observou-se um elevado percentual de indivíduos que referiram duas ou mais doenças crônicas, principalmente entre o sexo feminino $(55,1 \%)$. A prevalência de dependência nas atividades da vida diária foi elevada.

A distribuição dos participantes, de acordo com as demais variáveis do estudo, é mostrada na tabela 1.
Os dados da tabela 2 mostram a distribuição dos idosos do sexo feminino, segundo capacidade funcional e indicadores antropométricos de estado nutricional. A dependência em AIVDs foi significativamente mais prevalente nas mulheres com perímetro da panturrilha inferior ou superior. Já a dependência nas duas dimensões de capacidade funcional foi significativamente mais frequente apenas entre as mulheres que apresentaram perímetro da panturrilha inferior. A dependência funcional não variou de acordo com o IMC e AMB.

Os dados da tabela 3 mostram a distribuição dos idosos do sexo masculino, segundo capacidade funcional e indicadores antropométricos de estado nutricional. A dependência em ambas as dimensões de capacidade funcional foi significativamente mais prevalente nos homens que apresentaram IMC inadequado (baixo peso ou sobrepeso). A dependência funcional não variou segundo a $A M B$ e o perímetro da panturrilha.

A tabela 4 apresenta os resultados da análise ajustada (modelo logístico multinomial múltiplo) para capacidade funcional em relação às variáveis explanatórias do estudo no sexo feminino. A dependência em AIVDs foi positivamente associada ao perímetro da panturilha inferior. Não foram encontradas associações entre capacidade funcional e os outros indicadores de estado nutricional.

A tabela 5 apresenta os resultados da análise ajustada para capacidade funcional em relação às variáveis explanatórias do estudo no sexo masculino. A dependência em ABVDs e AIVDs foi positivamente ao IMC inadequado (baixo peso ou sobrepeso). Não foram encontradas associações entre capacidade funcional e os outros indicadores de estado nutricional.

Tabela 1. Características descritivas da população do estudo (Lafaiete Coutinho-BA, Brasil, 2012).

\begin{tabular}{|c|c|c|c|c|c|c|c|}
\hline \multirow{2}{*}{ Variáveis } & \multirow{2}{*}{$\begin{array}{c}\% \\
\text { resposta }\end{array}$} & \multicolumn{4}{|c|}{ Feminino Masculino } & \multicolumn{2}{|c|}{ Todos } \\
\hline & & $\mathrm{n}$ & $\%$ & $\mathrm{n}$ & $\%$ & $\mathrm{n}$ & $\%$ \\
\hline Número de doenças crônicas & 95,9 & & & & & & \\
\hline Nenhuma & & 20 & 12,0 & 42 & 30,9 & 62 & 20,5 \\
\hline Uma & & 55 & 32,9 & 48 & 35,3 & 103 & 34 \\
\hline Duas ou mais & & 92 & 55,1 & 46 & 33,8 & 138 & 45,5 \\
\hline Hospitalização & 99,4 & & & & & & \\
\hline Nenhuma & & 128 & 74,4 & 109 & 76,8 & 237 & 75,5 \\
\hline Uma ou mais vezes & & 44 & 25,6 & 33 & 23,2 & 77 & 24,5 \\
\hline Quedas & 99,4 & & & & & & \\
\hline Sim & & 56 & 32,4 & 25 & 17,7 & 81 & 25,8 \\
\hline Não & & 117 & 67,6 & 116 & 82,3 & 233 & 74,2 \\
\hline Atividade física & 98,1 & & & & & & \\
\hline Insuficientemente ativo & & 80 & 47,3 & 68 & 48,2 & 148 & 47,7 \\
\hline Ativo & & 89 & 52,7 & 73 & 51,8 & 162 & 52,3 \\
\hline Tabagismo & 99,7 & & & & & & \\
\hline Nunca fumou & & 103 & 59,6 & 30 & 21,1 & 133 & 42,2 \\
\hline Ex-fumante & & 63 & 36,4 & 84 & 59,2 & 147 & 46,7 \\
\hline Fumante & & 7 & 4,0 & 28 & 19,7 & 35 & 11,1 \\
\hline Capacidade funcional & 97,1 & & & & & & \\
\hline Independente & & 60 & 35,9 & 70 & 50,0 & 130 & 42,4 \\
\hline Dependente em AIVDs & & 79 & 47,3 & 47 & 33,6 & 126 & 41,0 \\
\hline Dependente em ABVDs e AIVDs & & 28 & 16,8 & 23 & 16,4 & 51 & 16,6 \\
\hline
\end{tabular}


Tabela 2. Associação entre indicadores antropométricos e capacidade funcional em idosos do sexo feminino (Lafaiete Coutinho-BA, Brasil, 2012).

\begin{tabular}{|c|c|c|c|c|c|c|}
\hline \multirow[t]{2}{*}{ Variáveis } & \multirow{2}{*}{\begin{tabular}{|c|} 
Referência* \\
$\%$
\end{tabular}} & \multicolumn{2}{|c|}{$\begin{array}{c}\text { Dependência } \\
\text { em AIVDs }\end{array}$} & \multicolumn{2}{|c|}{$\begin{array}{l}\text { Dependência em } \\
\text { ABVDs e AIVDs }\end{array}$} & \multirow[t]{2}{*}{ p-valor } \\
\hline & & $\%$ & OR (IC95\%) & $\%$ & OR (IC95\%) & \\
\hline \multicolumn{7}{|l|}{ IMC } \\
\hline Baixo peso & 29,4 & 58,8 & $1,53(0,61-3,82)$ & 11,8 & $1,04(0,26-4,09)$ & \multirow{3}{*}{0,654} \\
\hline Adequado & 37,1 & 48,6 & 1 & 14,3 & 1 & \\
\hline Sobrepeso & 41,8 & 41,8 & $0,76(0,35-1,65)$ & 16,4 & $1,02(0,35-2,94)$ & \\
\hline \multicolumn{7}{|l|}{ AMB } \\
\hline$\leq P_{25}$ & 30,8 & 51,3 & $1,40(0,53-3,73)$ & 17,9 & $1.33(0,37-4,83)$ & \\
\hline$>P_{25} a<P_{75}$ & 37,8 & 46,3 & 1 & 15,9 & 1 & 0,953 \\
\hline$\geq P_{75}$ & 38,1 & 45,2 & $1,03(0,46-2,37)$ & 16,7 & $0,96(0,32-2,88)$ & \\
\hline \multicolumn{7}{|l|}{$\begin{array}{c}\text { Perímetro da } \\
\text { panturrilha }\end{array}$} \\
\hline$\leq P_{25}$ & 12,8 & 59,0 & $7,89(2,48-25,42)$ & 28,2 & $13,2(2,96-58,92)$ & \\
\hline$>P_{25} a<P_{75}$ & 36,6 & 48,8 & 1 & 14,6 & 1 & 0,001 \\
\hline$\geq P_{75}$ & 57,1 & 33,3 & $2,29(1,01-5.15)$ & 9,5 & $2,40(0,69-8,40)$ & \\
\hline
\end{tabular}

IMC: índice de massa corporal. AMB: área muscular do braço.* Independente.

Tabela 3. Associação entre indicadores antropométricos e capacidade funcional em idosos do sexo masculino (Lafaiete Coutinho-BA, Brasil, 2012).

\begin{tabular}{|c|c|c|c|c|c|c|}
\hline \multirow[t]{2}{*}{ Variáveis } & \multirow{2}{*}{\begin{tabular}{|c|} 
Referência* \\
$\%$ \\
\end{tabular}} & \multicolumn{2}{|c|}{$\begin{array}{l}\text { Dependência } \\
\text { em AIVDs }\end{array}$} & \multicolumn{2}{|c|}{$\begin{array}{c}\text { Dependência em } \\
\text { ABVDs e AIVDs }\end{array}$} & \multirow[t]{2}{*}{$p$-valor } \\
\hline & & $\%$ & OR (IC95\%) & $\%$ & OR (IC95\%) & \\
\hline \multicolumn{7}{|l|}{ IMC } \\
\hline Baixo peso & 39,6 & 39,6 & $1,89(0,81-4,41)$ & 20,8 & $9,47(1,88-47,71)$ & \multirow{3}{*}{0,006} \\
\hline Adequado & 63,2 & 33,3 & 1 & 3,5 & 1 & \\
\hline Sobrepeso & 44,8 & 27,6 & $1,17(0,41-3,30)$ & 27,6 & $11,08(2,08-59,09)$ & \\
\hline \multicolumn{7}{|l|}{ AMB } \\
\hline$\leq P_{25}$ & 36,4 & 36,4 & $2,50(0,79-7,86)$ & 27,3 & $2,14(0,63-7,26)$ & \\
\hline$>P_{25} a<P_{75}$ & 52,2 & 37,7 & 1 & 10,1 & 1 & 0,105 \\
\hline$\geq P_{75}$ & 57,1 & 22,9 & $1,81(0,69-4,73)$ & 20,0 & $0,56(0,17-1,81)$ & \\
\hline \multicolumn{7}{|l|}{$\begin{array}{c}\text { Perímetro da } \\
\text { panturrilha }\end{array}$} \\
\hline$\leq P_{25}$ & 37,1 & 40,0 & $2,05(0,70-6,0)$ & 22,9 & $1,46(0,44-4,89)$ & \\
\hline$>P_{25} a<P_{75}$ & 55,4 & 33,8 & 1 & 10,8 & 1 & 0,255 \\
\hline$\geq P_{75}$ & 51,4 & 27,0 & $1,61(0,46-2,95)$ & 21,6 & $0,46(0,14-1,47)$ & \\
\hline
\end{tabular}

IMC: índice de massa corporal. AMB: área muscular do braço . ${ }^{*}$ Independente.

Tabela 4. Análise ajustada entre indicadores antropométricos de estado nutricional e capacidade funcional entre indivíduos do sexo feminino (Lafaiete Coutinho-BA, Brasil, 2012).

\begin{tabular}{c|c|c|c|c|c|c}
\hline \multirow{2}{*}{ Variável } & \multicolumn{2}{|c|}{ Dependência em AIVDs } & \multicolumn{3}{c}{$\begin{array}{c}\text { Dependência em ABVDs e } \\
\text { AIVDs }\end{array}$} \\
\cline { 2 - 7 } & OR $^{*}$ & IC95\% & p-valor & OR* & IC95\% & p-valor \\
\hline IMC & & & & & & \\
\hline Baixo peso & 2,00 & $0,61-6,57$ & 0,252 & 1,24 & $0,22-7,07$ & 0,812 \\
\hline Adequado & 1 & & & 1 & & \\
\hline Sobrepeso & 0,95 & $0,37-2,42$ & 0,912 & 1,72 & $0,45-6,62$ & 0,429 \\
\hline AMB & & & & & & \\
\hline$\leq P_{25}$ & 0,92 & $0,27-3,08$ & 0,890 & 0,64 & $0,12-3,38$ & 0,602 \\
\hline$>P_{25} \mathrm{a}_{75}$ & 1 & 1 & & 1 & 1 & \\
\hline$\geq P_{75}$ & 1,07 & $0,40-2,88$ & 0,894 & 0,72 & $0,19-2,77$ & 0,635 \\
\hline $\begin{array}{c}\text { Perímetro da } \\
\text { panturrilha }\end{array}$ & & & & & & \\
\hline$\leq \mathrm{P}_{25}$ & 5,77 & $1,20-27,61$ & 0,028 & 5,70 & $0,78-41,69$ & 0,087 \\
\hline$>\mathrm{P}_{25} \mathrm{a}<\mathrm{P}_{75}$ & 1 & 1 & & 1 & 1 & \\
\hline$\geq \mathrm{P}_{75}$ & 2,12 & $0,83-5,43$ & 0,118 & 1,90 & $0,47-7,70$ & 0,368 \\
\hline
\end{tabular}

* Ajustada por idade, número de doenças crônicas, hospitalizaçăo, quedas, atividade física e tabagismo.
Tabela 5. Análise ajustada entre indicadores antropométricos de estado nutricional e capacidade funcional entre indivíduos do sexo masculino (Lafaiete Coutinho-BA, Brasil, 2012).

\begin{tabular}{|c|c|c|c|c|c|c|}
\hline \multirow[t]{2}{*}{ Variável } & \multicolumn{3}{|c|}{ Dependência em AIVDs } & \multicolumn{3}{|c|}{$\begin{array}{l}\text { Dependência em ABVDs e } \\
\text { AIVDs }\end{array}$} \\
\hline & $\mathrm{OR}^{*}$ & IC95\% & p-valor & $\mathrm{OR}^{*}$ & IC95\% & p-valor \\
\hline \multicolumn{7}{|l|}{ IMC } \\
\hline Baixo peso & 2,42 & $0,85-6,85$ & 0,096 & 11,36 & $2,02-63,72$ & 0,006 \\
\hline Adequado & 1 & 1 & & 1 & 1 & \\
\hline Sobrepeso & 1,20 & $0,34-4,25$ & 0,779 & 22,06 & $3,24-150,34$ & 0,002 \\
\hline \multicolumn{7}{|l|}{ AMB } \\
\hline$\leq P_{25}$ & 3,01 & $0,69-13,07$ & 0,141 & 1,51 & $0,31-7,36$ & 0,611 \\
\hline$>P_{25} a<P_{75}$ & 1 & 1 & & 1 & 1 & \\
\hline$\geq P_{75}$ & 2,03 & $0,64-6,44$ & 0,230 & 0,37 & $0,09-1,49$ & 0,161 \\
\hline \multicolumn{7}{|l|}{$\begin{array}{l}\text { Perímetro da } \\
\text { panturrilha }\end{array}$} \\
\hline$\leq \mathrm{P}_{25}$ & 2,29 & $0,60-8,65$ & 0,223 & 1,20 & $0,29-4,91$ & 0,798 \\
\hline$>P_{25} a<P_{75}$ & 1 & 1 & & 1 & 1 & \\
\hline$\geq P_{75}$ & 1,23 & $0,41-3,68$ & 0,707 & 0,47 & $0,13-1,65$ & 0,238 \\
\hline
\end{tabular}

* Ajustada por idade, número de doenças crônicas, hospitalização, quedas, atividade física e tabagismo.

\section{DISCUSSÃO}

Na população investigada verificou-se que boa parte dos idosos é dependente para AIVDs, achado superior aos encontrados em outro estudo que utilizou escala semelhante e observou prevalência de 28,8\% de dependência nessas atividades em população de outra região do país ${ }^{20}$. Quanto à dependência em ABVDs, a prevalência encontrada foi baixa, se comparada a outros estudos realizados no Brasil que verificaram prevalências entre $34,8 \%$ e 40,9\% $\%^{21,22}$.

Observou-se que uma parcela grande da população apresentava pelo menos uma doença crônica. Esse achado é bastante preocupante, pois foi mostrado que a presença de doenças é um dos principais agravantes para incapacidade em idosos, assim como uma condição nutricional inadequada pode favorecer o acometimento de um número maior de doenças crônicas ou vice-versa ${ }^{23}$

Evidenciou-se no presente estudo que houve diferença entre os sexos quanto aos indicadores de estado nutricional que se associaram a capacidade funcional. Resultado semelhante foi encontrado em outro estudo, onde mudanças na composição corporal associadas à idade foram diferentes em homens e mulheres ${ }^{24}$.

Para o sexo feminino o único indicador de estado nutricional que teve associação com a capacidade funcional, independentemente das variáveis de ajuste, foi o perímetro da panturrilha. Observou-se que indivíduos do sexo feminino classificados como inferior $\left(\leq \mathrm{P}_{25}\right)$ apresentaram probabilidade quase seis vezes maior $(O R=5,77 ; p=0,028)$ de ser dependente na realização das AIVDs. De acordo com a Organização Mundial da Saúde ${ }^{4}$ o perímetro da panturrilha é o indicador antropométrico mais sensível em idosos para indicar alterações de massa magra que ocorre com o envelhecimento; isso pode explicar a associação apenas com as AIVDs, tendo em vista que esta dimensão da capacidade funcional envolve atividades que exigem mais da função muscular comparada as ABVDs.

Para o sexo masculino apenas o IMC se associou com capacidade funcional, independentemente da idade, número de doenças crônicas, hospitalização, ocorrência de quedas, atividade física e tabagismo. Tanto aqueles idosos com baixo peso, quanto os com sobrepeso, apresentaram maior probabilidade para desenvolvimento de dependência funcional nas ABVDs e AIVDs.

Esse achado é importante se for levado em conta que alterações na composição da massa corporal são frequentes entre idoso. Em um estudo realizado com a população idosa de Havana, Cuba, observou-se 
que a probabilidade de baixo peso aumentou progressivamente com o aumento da idade ${ }^{25}$.

A diferença quanto os indicadores antropométricos preditores de capacidade funcional em idosos diverge de um estudo realizado por Felix e Souza ${ }^{26}$ com idosos institucionalizados, que verificou não haver diferença entre os sexos quanto à utilização do IMC como preditor de estado nutricional. Isso sugere que preditores antropométricos de capacidade funcional utilizados para avaliação geriátrica de idosos institucionalizados podem não ser os mais adequados para idosos residentes em comunidade. Setiati et al. ${ }^{27}$ aponta a necessidade de se utilizar valores e indicadores antropométricos de estado nutricional específicos a cada sexo. De acordo com esses autores, na investigação do estado nutricional em idosos, deve-se buscar minimizar o máximo possível a presença de viés, já que o envelhecimento apresenta características bastante peculiares.

Essas diferenças podem resultar de características específicas dos indicadores. A utilização do IMC em idosos é dificultada devido à diminuição da estatura, redução da massa corporal magra, acúmulo de gordura visceral, e redução de água no organismo. Sendo essas alterações mais evidentes no sexo masculino, o índice acaba por refletir maior comprometimento neste grupo ${ }^{28}$.

Entretanto, o perímetro da panturrilha foi apontado como um dos indicadores de estado nutricional que apresentou alterações mais relevantes com o avançar da idade na população idosa feminina ${ }^{29}$.

Os resultados obtidos precisam ser avaliados com cautela em função de possíveis limitações do estudo. Devem-se considerar aquelas relativas ao tipo de desenho de estudo utilizado - transversal - que avalia simul- taneamente variáveis relativas ao efeito de interesse e aos seus fatores associados. Portanto, não se pode descartar a hipótese de causa reversa.

Apesar destas limitações, o estudo avaliou um contingente expressivo de pessoas, o que permitiu estimativas confiáveis quanto às variáveis estudadas. Os resultados obtidos representam informação útil para a gestão em saúde, servindo como subsídio para as políticas públicas de intervenção em saúde do idoso.

\section{CONCLUSÃO}

Diante dos resultados encontrados é possível concluir que o indicador antropométrico de estado nutricional que melhor se associa a capacidade funcional em idosos residentes em comunidade é dependente do sexo, com o perímetro da panturrilha, sendo melhor preditor em mulheres, da mesma forma como é o IMC em homens. Reforça-se a importância da utilização da antropometria na prática clínica ou em intervenções comunitárias como instrumento de triagem para dependência funcional e fragilidade.

\section{AGRADECIMENTOS}

J.C.C.S.J. recebeu bolsa de Iniciação Científica da Universidade Estadual do Sudoeste da Bahia, BA, Brasil. Os autores agradecem a Secretaria Municipal de Saúde de Lafaiete Coutinho, BA, Brasil e os idosos que participantes do estudo.

Todos os autores declararam não haver qualquer potencial conflito de interesses referente a este artigo.

\section{REFERÊNCIAS}

1. Veras R. Population aging today: demands, challenges and innovations. Rev Saúde Publica 2009;43(3):548-54.

2. Ávila-Funes JA, Gray-Donald K, Payette H. Association of nutritional risk and depressive symptoms with physical performance in the elderly: the Quebec longitudinal study of nutrition as a determinant of successful aging (NuAge). J Am Coll Nutr. 2008;27(4):492-8.

3. Borson S. Cognition, Aging and Disabilities: Conceptual Issues. Phys Med Rehabil Clin N Am. 2010;21(2):375-82.

4. World Health Organization. Expert Committee on Physical Status: the use and interpretation of anthropometry physical status. Switzerland; 1995.

5. Garcia PA, Dias JMD, Dias RC, Santos P, Zampa C. A study on the relationship between muscle function, functional mobility and level of physical activity in community-dwelling elderly. Rev Bras Fisioter 2011;15(1):15-22.

6. Albala C, Lebrão ML, Díaz EML, Ham-Chande R, Hennis AJ, Palloni A et al. Encuesta Salud, Bienestar y Envejecimiento (SABE): metodología de la encuesta y perfil de la población estudiada. Rev Panam Salud Pública. 2005;17:307-22.

7. Katz S, Ford AB, Moskowitz RW, Jackson BA,Jaffe MW. Studies of illness in the aged. The index of ADL: a standardized measure of biological and psychosocial function. JAMA. 1963;185:914-9.

8. Lawton MP, Brody EM. Assessment of older people: self-maintaining and instrumental activities of daily living. Gerontologist. 1969;9(3):179-86.

9. Hoeymans N, Feskens EJM, van den Bos GAM, Kromhout D. Measuring functional status: crosssectional and longitudinal associations between performance and self-report (Zuthen Elderly Study 1990-1993). J Clin Epidemiol. 1996;49:1103-10.

10. Rodrigues MAP, Facchini LA, Thumé E, Maia F. Gender and incidence of functional disability in the elderly: a systematic review. Cad Saúde Publica. 2009;25 (3):S464-76.

11. Frisancho AR. New standards of weight and body composition by frame size and height for assessment of nutritional status of adults and the elderly. Am J Clin Nutr. 1984;40:808-19.

12. Callaway WC, Chumlea WC, Bouchard C, Himes JH, Lohman TG, Martin AD, et al. Circumferences. In: Lohman TG, Roche AF, Martorell R, editors. Anthropometric Standardization Reference Manual. Champaign: Human Kinetics. 1988. p.39-54.

13. Harrison GG, Buskirk RE, Carter JEL, Johnston FE, Lohman TG, Pollock ML, et al. Skinfold thicknesses. In: Lohman TG, Roche AF, Martorell R, editors. Anthropometric Standardization Reference Manual. Champaign: Human Kinetics. 1988. p.55-70.

14. Heymsfield SB, McManus C, Smith J, Stevens V, Nixon DW. Anthropometric measurements of muscle mass: revised equations for calculating bone-free arm muscle area. Am J Clin Nutr. 1982;36:680-90.

15. American Academy of Family Physicians, American Dietetic Association, National Council on the
Aging. Nutrition screening e intervention resources for healthcare professionals working with older adults. Nutrition Screening Initiative. Washington: American Dietetic Association; 2002. Avaliable from: <http://www.eatright.org/cps/rde/xchg/ada/hs.xsl/nutrition_nsi_ENU_HTML.htm>. [2011 set 21].

16. Craig CL, Marshall AL, Sjostrom M et al. International Physical Activity Questionnaire: 12-country reliability and validity. Med Sci Sports Exerc. 2003; 35:1381-95.

17. Hallal PC, Victora CG, Wells JC, Lima RC. Physical inactivity: prevalence and associated variables in Brazilian adults. Med Sci Sports Exerc. 2003;35:1894-900.

18. Campos MTFS, Monteiro JBR, Ornelas APRC. Fatores que afetam o consumo alimentar e a nutrição do idoso. Rev Nutr. 2000;13(3):157-65

19. Campos MAG, Pedroso ERP, Laumonier JA, Colosimo EA, Abrantes MM. Estado nutricional e fatores associados em idosos. Rev Assoc Med Bras. 2006; 52(4):214-21.

20. Del Duca GF, Silva MC,Hallal PC. Incapacidade funcional para atividades básicas e instrumentais da vida diária em idosos. Rev Saude Publica. 2009;43(5):796-805.

21. Nunes DP, Nakatani AYK, Silveira EA, Bachion MM, Souza MR. Capacidade funcional, condições socioeconômicas e de saúde de idosos atendidos por equipes de Saúde da Família de Goiânia (GO, Brasil). Ciênc. Saúde Colet. 2010;15(6): 2887-98.

22. Nakatani AYK, Silva LB,Bachion MM, Nunes DP. Capacidade funcional em idosos na comunidade $e$ propostas de intervenções pela equipe de saúde. Rev Eletr Enf. 2009;11(1):144-50.

23. Costa e Silva MD, Guimarães HA, Trindade Filho EM, Andreoni S, Ramos LR. Fatores associados à perda funcional em idosos residentes no município de Maceió, Alagoas. Rev Saúde Publica. 2011;45(6):1137-44.

24. Lopes ACS, Santos LC, Lima-Costa MF, Caiaffa WT. Nutritional factors associated with chronic noncommunicable diseases - the Bambuí Project: a population-based study. Cad Saúde Pública. 2011;27(6):1185-91.

25. Coqueiro RS, Barbosa AR, Borgatto AF. Nutritional status, health conditions and socio-demographic factors in the elderly of havana, cuba: data from sabe survey. J Nutr. 2010;14(10):803-8.

26. Félix LN, Souza EMT. Avaliação nutricional de idosos em uma instituição por diferentes instrumentos. Rev Nutr. 2009;22(4) 571-80.

27. Setiati S, Istanti R, Andayani R, Kuswardhani RAT, Aryana IGPS, Putu ID et al. Cut-off of Anthropometry Measurement and Nutritional Status Among Elderly Outpatient in Indonesia:Multi-centre Study. Acta Med Indones-Indones J Intern Med. 2010;42(4):224-30.

28. Garcia ANM, Romani SAM, Lira PIC. Indicadores antropométricos na avaliação nutricional de idosos: um estudo comparativo. Rev Nutr. 2007;20(4):371-8.

29. Krause MP, Buzzachera CF, Hallage T, Santos ECR, Silva SG. Alterações morfológicas relacionadas à idade em mulheres idosas. Rev Bras Cineantropom Desempenho Hum. 2006;8(2):73-7.DISCUSSÃO 